\title{
Notes on Detrital Remanent Magnetization of Sediments
}

\author{
By Takesi Nagata \\ Department of Geology, University of Pittsburgh \\ (Read May 25, 1962 ; Received May 26, 1962)
}

\begin{abstract}
A mathematical formula representing the resultant magnetic moment of detrital remanent magnetization (DRM) of sediments, originally proposed by the writer, is revised based on a more reasonable assumption. Using the revised expression, the inclination error of DRM is satisfactorily interpreted as due to the effect of the spheroidal form of magnetized particles in the sediments.
\end{abstract}

\section{Introduction}

The mechanism of formation of detrital remanent magnetization (DRM) of sedimentary rocks in still water has been fairly well understood. The mechanism was mathematically expressed by Nagata et al (1943), and the mathematical formula has been proved to fit the experimental results well $(1953,1961)$. Effects of shape of depositing magnetic particles and the inclination of the bedding plane upon the direction of DRM have also been studied by King (1955), Griffith et al $(1957,1960)$ and by Ising (1943) and Granar (1958) experimentally and theoretically.

Thus, the mechanism of formation of DRM seems to be understood qualitatively, when applied to sediments assumed to have been deposited in still water.

It seems however that an inappropriate assumption was adopted in the mathematical calculation made by Nagata et al. One of the aims of this note is to revise the original mathematical expression on a more reasonable assumption. On the other hand, the fact that the inclination of DRM is always smaller than that of an affecting magnetic field has long been noticed. This deviation of the inclination, called the inclination error, has been considered to be caused by the non-spherical shape of magnetized particles. However, the inclination error has not yet been fully understood on the basis of dynamics of depositing particles. A part of the aim of this note is to clarify the inelination error quantitatively based on the revised expression of DRM characteristics.

\section{DRM of Spherical Particle Deposits in Still Water onto Horizontal Beds}

The fundamental idea used in the calculations in this note is the same as the original one. A rotation of a spherical magnet, $\sigma$ in moment, in a magnetic field $H$ in still water is expressed as

$$
\lambda \frac{d \theta}{d t}+\sigma H \sin \theta \simeq 0
$$


where $\theta$ denotes the angle between the magnetic moment and the field direction; and $\lambda$ is the viscosity coefficient for rotation of the particle. The solution of (1) is given as

$$
\tan \theta / 2=\tan \left(\theta_{0} / 2\right) \exp \left(-\frac{\sigma H}{\lambda} t\right),
$$

where $\theta_{0}$ means the initial value of $\theta$ at $t=0$. In this example, $t$ indicates the time for deposition of a spherical particle from the water surface to the bedding plane at the bottom.

Since the direction of the magnetic moment is considered to be at random at $t=0$, the total resultant magnetic moment $J$ of a deposit of a large number of the particles at $t=t$ is derived from (3) as

$$
J=\frac{N \sigma}{2} \int_{0}^{\pi} \cos \theta \sin \theta_{0} d \theta_{0} .
$$

In the original expression ${ }^{(1)}, J$ was expressed as

$$
J=\frac{N \sigma}{\pi} \int_{0}^{\pi} \cos \theta d \theta_{0}=N \sigma \tanh \left(\frac{\sigma H}{2 \lambda} t\right),
$$

by assuming a uniform distribution of $\theta_{0}$ between 0 and $\pi$. Obviously, this assumption is not correct when the particles have the three dimensional freedom.

(3) leads to

$$
J=N \sigma\left[\operatorname{coth}\left(\frac{\sigma H}{\lambda} t\right)-\frac{\sigma H t}{\lambda} \operatorname{cosech}^{2}\left(\frac{\sigma H}{\lambda} t\right)\right],
$$

which gives $J=0$ at $t=0$, and $J=N \sigma$ at $t \rightarrow \infty$. The curve represented by (5) is compared with the curve given by (4) in Figure 1, where $J / N \sigma=D(x) \equiv \operatorname{coth} x-x \operatorname{cosech}^{2} x$ for (5)

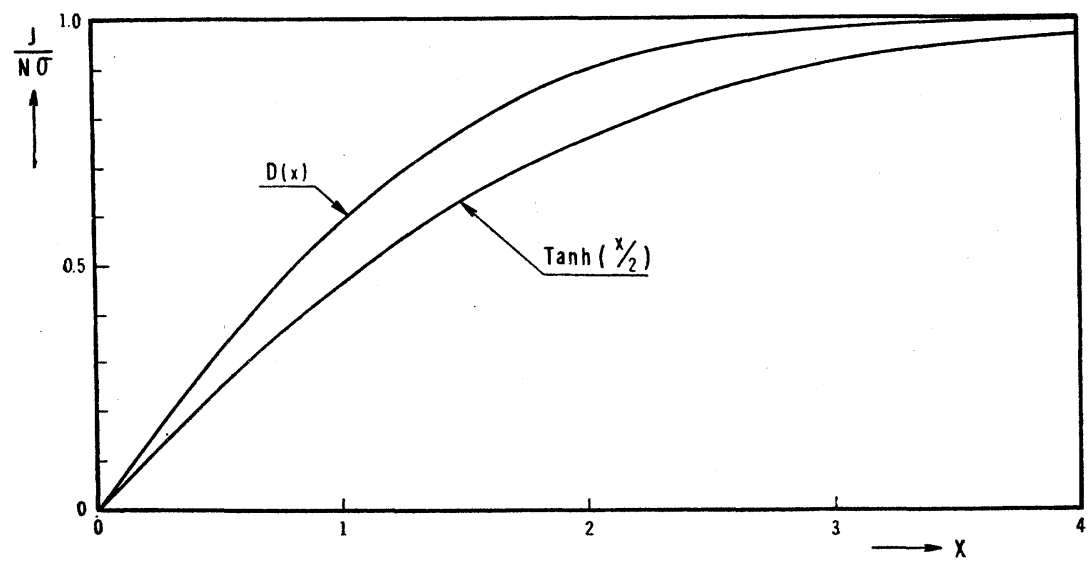

Fig. 1. Theoretical curves of resultant magnetic moment of DRM as a function of time or intensity of an affecting magnetic field. $\left(x=\frac{\sigma H}{\lambda} t\right)$

and $J / N \sigma=\tanh (x / 2)$ for (4), $x$ replacing $\sigma H t / \lambda$ in both cases. The increase in $J$ with time in the revised formula is definitely faster than in the original formula. However, the functions $D(x)$ defined here are expressed as 


$$
\begin{aligned}
& D(x) \simeq \frac{2}{3} x \quad \text { when } x \ll 1, \\
& D(x) \simeq 1-2 x e^{-2 x} \text { when } x \rightarrow \infty .
\end{aligned}
$$

Therefore, $D(x)$ can be approximated roughly by $\tanh (2 x / 3)$, which has characteristics expressed by

$$
\begin{aligned}
& \tanh (2 x / 3) \simeq \frac{2}{3} x \quad \text { for } x \ll 1, \\
& \tanh (2 x / 3) \simeq 1-2 e^{-\frac{4}{3} x} \text { for } x \rightarrow \infty .
\end{aligned}
$$

In a practical case of DRM of sediments, the depth $h$ of still water is related to $t$ as $h=\frac{m}{\nu} g t$, where $m, g$ and $\nu$ denote respectively the mass of a particle, the force of gravity and the viscosity coefficient for translation. Hence, $x=\sigma H t / \lambda$ or $\sigma H \nu h / \lambda m g$.

Although $\sigma, H$ and $t$ or $h$ are independently measurable in practical experiments, $\lambda$ or the ratio, $\nu / \lambda$ can be determined only by the artificial DRM experiment. Therefore, either $\mathrm{J}=N \sigma D(x)$ or $J=N \sigma \tanh (2 x / 3)$ can fit the experimental data. This may be the reason why the original expression can represent well the empirical relationship between $J$ and $H$ or between $J$ and $h$.

\section{Distorsion of the Distribution Function of Magnetic Orientation of Particles with Time}

With regard to the discussion on the origin of remanent magnetization of sedimentary rocks, the distribution function of magnetic orientation of magnetic particles will give certain information. For example, the natural remanent magnetization of a red sandstone may be attributed either to crystalline remanent magnetization (CRM) in situ or to the DRM of redeposited particles already magnetized. The distribution function of the magnetic orientation of individual particles may be different between the first and the second possibility.

The distribution function $f(\theta)$ of DRM in still water can be derived from (2) as a function of time $t$. Let us assume that the initial form of the distribution function at $t=0$ is expressed as

$$
f\left(\theta_{0}\right)=\frac{N}{2} \sin \theta_{0},
$$

where $\int_{0}^{\pi} f\left(\theta_{0}\right) d \theta_{0}=N$. The transformation of $f\left(\theta_{0}\right)$ to the distribution $f(\theta)$ at $t=t$ is derived by transformation of $f\left(\theta_{0}\right) d \theta_{0}$ to $f(\theta) d \theta$ according to the basic relation expressed by (2). Namely,

$$
f(\theta) d \theta=\frac{N}{2} \frac{\sin \theta}{e^{-x} \cos ^{2} \frac{\theta}{2}+e^{x} \sin ^{2} \frac{\theta}{2}} d \theta .
$$

Eq. (7) shows that $f(\theta)=\frac{N}{2} \sin \theta$ when $x=0($ i.e. $t=0$ ) and $f(\theta)=0$ except at $\theta=0$ when $x \rightarrow \infty$. On the other hand, $\int_{0}^{\pi} f(\theta) d \theta=N$, regardless of the value of $x$. Naturally,

$$
J=\int_{0}^{\pi} \sigma f(\theta) \cos \theta d \theta=N \sigma D(x) .
$$




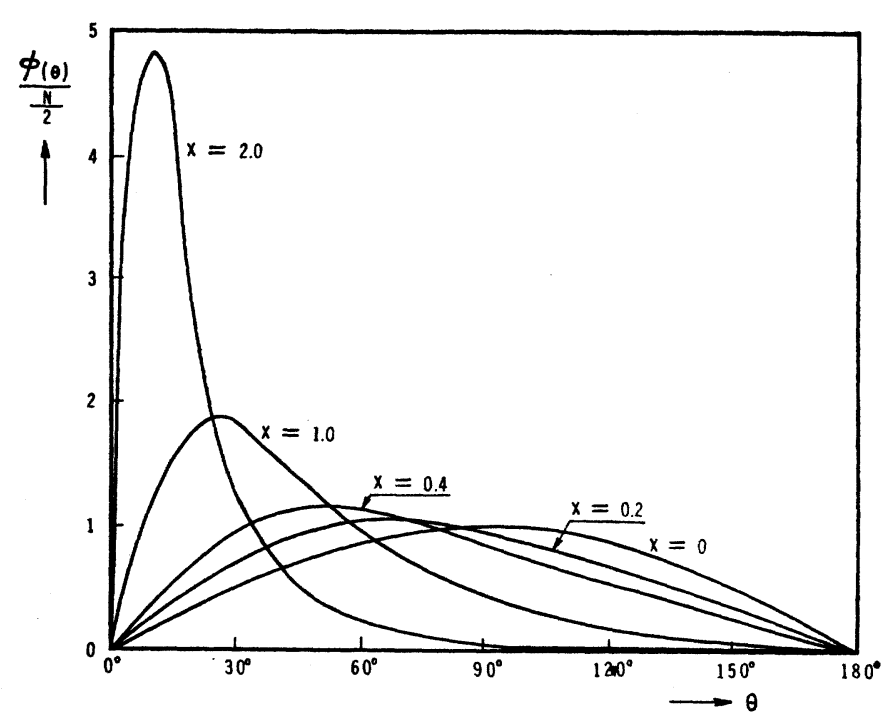

Fig. 2. Distribution function $\phi(\theta)$ of magnetic orientation of particles as a function of $x$.

Examples of the distorsion of the distribution function with an increase in $x$ are illustrated in Fig. 2.

Comparing Fig. 2 with Fig. 1, it is noticed that the distorsion of $f(\theta)$ towards the $H$ direction is appreciably large even in the case where $x=0.4$ which results in $J \simeq 0.26$ No. Also the largest amount of magnetic particles are within $\theta=30^{\circ}$ where $x=2$ when $J \simeq 0.9 N \sigma$. In the Narita-bed sandstone, $\alpha=J / N \sigma$ is estimated to be $0.1 \sim 0.2$ (Nagata et al, 1949). For the varved clay of New England (Johnson et al, 1948), $\alpha$ is $\frac{1}{4} \sim \frac{1}{3}$. Hence, the feature of distorsion of $f(\theta)$ in natural sediments will fall between the curve of $x=0.2$ (the corresponding $\alpha \simeq 0.13$ ) and the curve of $x=0.4(\alpha \simeq 0.26)$ in Fig. 2 . It is presumed, on the other hand, that CRM or the thermoremanent magnetism (TRM) of an assemblage of magnetic particles constituting of multidomains and polycrystals has a much more uniform magnetic orientation; or in other words, the distribution function $f(\theta)$ is much distorted in such a form similar to the case of $x=2$ or larger values of $x$.

It seems therefore that detailed studies on the distribution function of the natural remanent magnetization of sediments may give a criterion to distinguish their CRM origin from their DRM origin.

\section{A Theoretical Interpretation of the Inclination Error of DRM}

Since the first early experiments on the artificial deposition of sediments made by Nagata $e t a l^{(1)}$ and Johnson et al (1948), it has been noticed that the inclination of DRM of the sediments is smaller than that of an applied field by $10 \sim 20$ degrees. The inclination error is believed to be mainly attributable to the spheroidal shape of depositing particles, as discussed by Ising (1943), Granar(1958) and Nagata(1953), by refering to the characteristic of anisotropic magnetic susceptibility of the sediment.

King (1955) has carried out a systematic experiment to study the inclination error of 


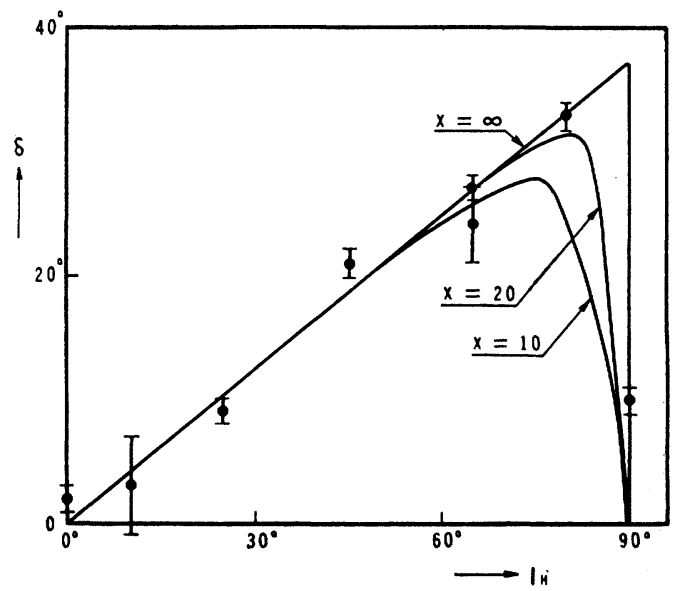

Fig. 3. The inclination error $\delta$ as a function of inclination $I_{I I}$ of the affecting magnetic field.

Full circles: empirical data. (after King)

Full lines: theoretical values. $(f=0.58)$

$\mathrm{DRM}$, and has determined the magnitude of the error $\delta$ as a function of the inclination $I_{H}$ of the affecting magnetic field, which is duplicated in Fig. 3. However, his interpretation of the $\delta \sim I_{H}$ relation is hardly understandable from the viewpoint of dynamics of depositing magnetic particles. The $\delta \sim I_{H I}$ relation must be explained clearly on the basis of the dynamics, because the dynamics are not as complex in the physical ideas underlying them. Let us consider somewhat idealized model such that a fraction $f$ of the magnetized particles is of spherical shape whereas the remaining portion $(1-f)$ are spheroidal, both hav-

ing the same magnetic moment $\sigma$.

Then, the total magnetic moment of the portion of spherical form at $t=t$ is given by (5) as

$$
\left.\begin{array}{l}
J_{0}=f N \sigma D(x), \quad x=\frac{\sigma H}{\lambda} \cdot t, \\
D(x)=\operatorname{coth} x-x \operatorname{cosech}^{2} x,
\end{array}\right\}
$$

where $J_{0}$ is parallel to $\mathrm{H}$. The spheroidal particles may be assumed to have their magnetization along the major axis (in case of prolate ellipsoid) or within the major axial plane (for an oblate shape). The major axis or the major axial plane may be set parallel to the bedding plane. Hence these particles are considered to have the twodimensional freedom around their vertical axis. Then the total magnetic moment of the portion of spheroidal form at $t=t$ is given by (4) as

$$
J^{\prime}=(1-f) N \sigma \tanh \left(\frac{x \cos I_{H_{-}}}{2}\right)
$$

where $I_{I I}$ denotes the inclination of the affecting magnetic field $H$. Obviously $J^{\prime}$ is parallel to the bedding plane which is horizontal.

Defining the inclination error $\delta$ by

$$
\delta=I_{H}-I_{0}
$$

where $I_{0}$ denotes the inclination of the total resultant magnetic moment $J=J_{0}+J^{\prime}$, we get

$$
J_{0} \sin \delta=J^{\prime} \sin I_{0}
$$

Putting (8) and (9) into (11), we get

$$
\tan I_{0}=\frac{f \sin I_{I I}}{f \cos I_{H}+(1-f) \tan h\left(x \cos I_{I I} / 2\right) / D(x)} .
$$

When $x$ is small, namely, when $x \ll 1$, (12) is approximated as 


$$
\tan I_{0}=\frac{4 f}{3+f} \tan I_{H}
$$

On the contrary, when $x \rightarrow \infty$ (12) becomes

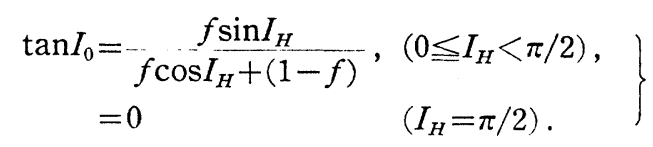

(13) is valid with an error less than $5 \%$ for the range of $x \leqq 0.6$ and with an error less than $10 \%$ for the range of $x \leq 0.8$. $\mathrm{King}^{(3)}$ has assumed a priori that $J_{0} \propto f_{H}$ and $J^{\prime} \cos (1-f) H \cos I_{H}$ and has derived a relation that $\tan I_{0}=f \tan I_{H}$.

The relationship (13) between $I_{0}$ and $I_{H}$ for small values of $x$ obtained in the present paper is of the same mathematical form as that proposed by King. Hence, King's

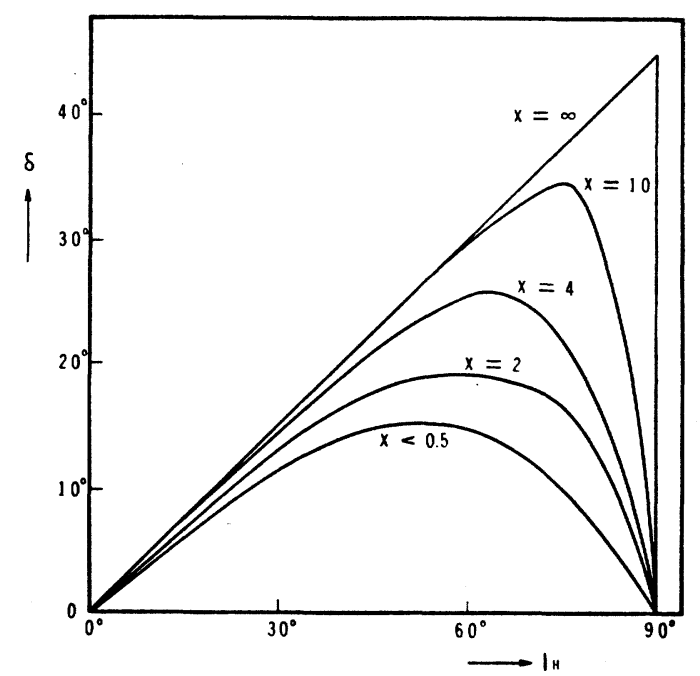

Fig. 4. Theoretical value of the inclination error $\delta$ as a function of the inclination $I_{H}$ of the applied magnetic field. $(f=0.5)$ formula may be considered as an approximate expression of the $I_{0} \sim I_{H}$ relation for small values of $x$. Fig. 4 illustrates an example of the relation for $f=0.5$ for various values of $x$. According to increase in $x=\sigma H t / \lambda$, the inclination error $\delta$ increases, approaching an asymptotic form, expressed by $\tan \delta=(1-f) \sin I_{H} /\left[f+(1-f) \cos I_{H}\right]$. .

King's experiments were done under steady state conditions $(t \simeq \infty)$. This steady state corresponds to the condition where $\delta$ is near the asymptotic value. Theoretical curves of $\delta$ obtained by (12), (13) and (14) are shown in Fig. 3, where $f$ is assumed to be 0.58 . The agreement between the experimental values and the theoretical curve is satisfactorily good provided that $x$ is about 20 or a little larger.

We may conclude therefore that the observed inclination error can be interpreted quantitatively as due to the elongation of the grains along the direction of their magnetization. Griffith et al (1960) have attributed the error to the effect of rotation of particles around the axis of horizontal component of the magnetic field. This effect could take place to some extent. However, the satisfactory agreement of the present theoretical curve with the empirical data may indicate that the main cause of the inclination error is the spheroidal shape of the magnetic particles, as already argued (Nagata, 1961) in connexion with anisotropic magnetic susceptibility.

\section{The Intensity of DRM of a Mixture of Spherical and Spheroidal Magnetic Grain}

Since the idealized model of magnetized grains consisting of a fraction $f$ of 
spherical shape and the remaining $(1-f)$ portion of spheroidal configuration is proved to well explain the observed inclination error, the dependency of intensity $J$ of DRM upon deposition time $t$ and applied magnetic field $H$ must be revised based on this model.

From (8) and (9), $J$ in such a case is expressed as

$$
J=N \sigma f\left[D^{2}(x) \sin ^{2} I_{H I}+\left\{D(x) \cos I_{I I}+\frac{1-f}{f} \tan h\left(\frac{x \cos I_{H}}{2}\right)\right\}^{2}\right]^{\frac{1}{2}} .
$$

Examples of calculated $J(x)$ curves are illustrated in Fig. 5, where the $D(x)$ curve is

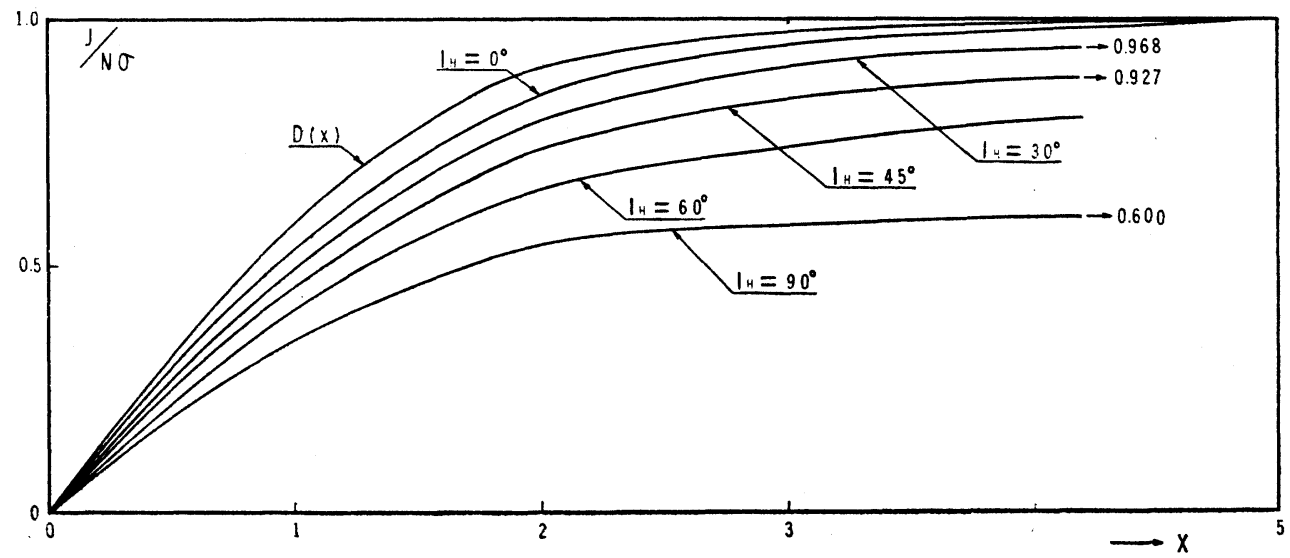

Fig. 5. Calculated examples of intensity of DRM of mixtures of spherical and spheloidal magnetic grains as dependent on magnetic field inclination $I_{H}$. $(f=0.6)$

also shown for comparison. Obviously, the $J(x)$ curves differ for different values of $I_{H}$ and the content of spheroidal grains $(1-f) . J$ at the final steady state $(x \rightarrow \infty)$, which is expressed as

$$
\begin{aligned}
J(x=\infty) & =N \sigma f\left[1+\left(\frac{1-f}{f}\right)^{2}+2 \frac{1-f}{f}-\cos I_{H}\right]^{\frac{1}{2}} & I_{H}<\frac{\pi}{2}, \\
& =N \sigma f & I_{H}=\frac{\pi}{2} .
\end{aligned}
$$

is illustrated in Fig. 6 -(a). On the other hand, $J$ at the initial stage while $x \ll 1$ is illustrated in Fig. 6-(b).

As shown in Fig. 6-(a), the final steady value of $J$ is the same for a same value of $f$ or $1-f$. This would be obvious because the resultant magnetization at the final stage depends only on the mutual geometrical relation between the lines of magnetic force and the horizontal bed. However, the initial value of $J$ decreases markedly with increse in $f$ as shown in Fig. 6-(b), which indicates that the motion of spheroidal grains affected by a magnetic field is appreciably slower than that of spherical ones.

It seems thus an interpretation of intensity of DRM of sediments is considerably complicated, if the model of a mixture of spherical and spheroidal magnetic grains is adopted. The present theory suggests that $J$ at a quasi-steady state should decrease with increase in $I_{H}$. The tendency that $J$ of artificial DRM is apt to decrease with increase in $I_{H}$ has been only qualitatively noticed. It seems necessary therefore that 

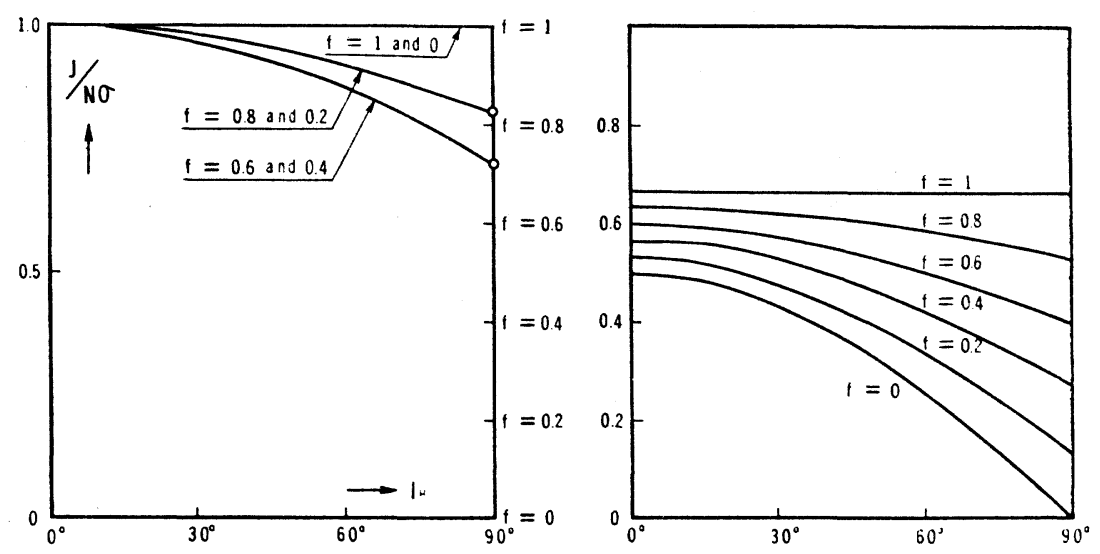

Fig. 6. Final and initial values of intensity of DRM

(a) Final value

$$
\frac{J}{N \sigma} \text { at } x \rightarrow x
$$

(b) Initial value

$$
\frac{J}{N \sigma x} \text { at } \mathrm{x} \rightarrow 0
$$

a quantitative study on $J$ as a function of $I_{l l}$ systematically be carried out together with measurements of the inclination error and susceptibility anisotropy of the same specimens.

In concluding, the mechanism of DRM of sediments must be thoroughly re-examined on a quantitative experimental basis to deal with their DRM for palaeomagnetic purposes. It seems likely that the two-constituent model of sediments, discussed in the present note, will be one of the basic elements in such a study.

\section{Acknowledgement}

This paper is a part of the reports of the earth-magnetism research program in Department of Geology, Lniversity of Pittsburgh, which is undertaken with the research grand NSF-G 21542, granted from the National Science Foundation, and with the research fund granted from the Claude Worthington Benedum Foundation. The author's thanks are due to Dr. A.F. Frederickson and Mr. Paul Benedum for their general support to this research program.

\section{References}

Granar, A. (1958) Ark. Geofys., 3, No. 1.

Griffith, D.H., King, R.F., Rees, A.I. and Wright A.E. (1960) Proc. Roy. Soc. London, A. 256, 359. Griffith, D.H., King, R.F. and Wright, A.E. (1957) Adv. Phys., 6, 306.

Ising, G. (1943) Ark. Geofys., 29, A, No. 5.

Johnson, E.A., Murphy, T. and Torreson, O.W. (1948) Terr. Mag., 53, 349.

King, R.F. (1955) M.N.R.A.S. Geophys. Suppl., 7, 115.

Nagata, T. (1953) Rock Magnetism, First Ed. (Maruzen Co., Ltd., Tokyo) 198-200.

Nagata, T. (1961) Rock Magnetism, Revised Ed. (Maruzen Co., Ltd., Tokyo) 227-229.

Nagata, T., Hirao, K. and Yoshikawa, H. (1949) J. Geomag. Geoele., 1, 52.

Nagata, T., Rikitake, T. and Akasi, K. (1943) Bull. Earthq. Res. Inst., 21, 276. 